\title{
Kondo effect and spin quenching in high-spin molecules on metal substrates
}

\author{
D. Jacob, ${ }^{1, *}$ M. Soriano, ${ }^{2}$ and J. J. Palacios ${ }^{3}$ \\ ${ }^{1}$ Max-Planck-Institut für Mikrostrukturphysik, Weinberg 2, 06120 Halle, Germany \\ ${ }^{2}$ Departamento de Física de la Materia Condensada, Universidad Autónoma de Madrid, Madrid, Spain \\ ${ }^{3}$ Departamento de Física de la Materia Condensada, Instituto de Física de la Materia Condensada (IFIMAC), \\ and Instituto Nicolás Cabrera (INC), Universidad Autónoma de Madrid, Madrid, Spain
}

(Received 7 July 2013; published 18 October 2013)

\begin{abstract}
Using a state-of-the art combination of density functional theory and impurity solver techniques, we present a complete and parameter-free picture of the Kondo effect in the high-spin $(S=3 / 2)$ coordination complex known as manganese phthalocyanine adsorbed on the $\mathrm{Pb}(111)$ surface. We calculate the correlated electronic structure and corresponding tunnel spectrum and find an asymmetric Kondo resonance, as recently observed in experiments. Contrary to previous claims, the Kondo resonance stems from only one of three possible Kondo channels with origin in the Mn $3 d$ orbitals, its peculiar asymmetric shape arising from the modulation of the hybridization due to a strong coupling to the organic ligand. The spectral signature of the second Kondo channel is strongly suppressed as the screening occurs via the formation of a many-body singlet with the organic part of the molecule. Finally, a spin- $1 / 2$ in the $3 d$ shell remains completely unscreened due to the lack of hybridization of the corresponding orbital with the substrate, hence leading to a spin-3/2 underscreened Kondo effect.
\end{abstract}

DOI: 10.1103/PhysRevB.88.134417

PACS number(s): 75.50.Xx, 71.27.+a, 72.10.Fk, 74.55.+v

Whenever a magnetic atom or magnetic molecule is coupled to metallic electrodes, the conduction electrons are likely to screen its magnetic moment through the Kondo effect, ${ }^{1,2}$ which is signaled by Fano-Kondo line shapes in the conductance spectra. ${ }^{3}$ Although this usually occurs at low temperatures, it may have important consequences for possible applications of molecular magnets ${ }^{4}$ as ultimately miniaturized magnetic storage units or as prospective nanoscale spintronics devices. ${ }^{5}$ Alternatively, the Kondo effect could also serve as a sensor of magnetic state changes when the molecule is subjected to mechanical deformation ${ }^{6,7}$ or chemical changes ${ }^{8,9}$ without the necessity of applying a magnetic field, thus opening the door to novel applications of this quantum effect.

From a more fundamental point of view, atomic precision experimental control offers the possibility to study a wide range of electron correlation phenomena related to the Kondo effect. For example, the atomic-scale control of atoms or molecules adsorbed on metal surfaces or anchored to nanoscopic electrodes allows for a direct manipulation of the orbital hybridization and for a controlled tuning from the so-called underscreened to the overscreened Kondo effects, both regimes showing interesting non-Fermi-liquid behavior. ${ }^{10}$ Recently, underscreened Kondo effects have been reported for a $\mathrm{C}_{60}$ quantum dot molecule coupled to metal leads ${ }^{11}$ and for a $\mathrm{Co}(\text { tpy-SH })_{2}$ complex coupled to Au nanocontacts. ${ }^{7}$ The overscreened Kondo effect, on the other hand, has been very recently predicted to occur in Au nanocontacts hosting a single Co atom. ${ }^{12}$

Coordination complexes are of much interest in this regard. In particular, the family of planar organic molecules containing a transition metal (TM) center at its core are nicely suited for controlled experiments with scanning tunneling microscopy. The exchange-induced magnetic moment of the TM atom can be as high as $S=5 / 2$, but the strong coupling to the organic ligand usually quenches the spin into lower values. ${ }^{13}$ Crystal field theories can nicely explain the lowering of the high-spin state. ${ }^{14}$ This phenomenon can also be properly described by standard implementations of density functional theory (DFT), i.e., by an effective one-electron approximation. Similarly, DFT can also explain charge-transfer processes, typically between the TM and the surface, which can also quench the spin. However, often in these systems both one-body quenching and many-body screening processes coexist, making it very difficult to disentangle their respective contributions to the experimental signatures. ${ }^{15}$

Here, by treating both screening and quenching on the same footing, we elucidate the relevant mechanisms behind the experimental observations in a single manganese phthalocyanine $(\mathrm{MnPc})$ absorbed on the (111) surface of $\mathrm{Pb}$ in the normal (i.e., not superconducting) phase. ${ }^{16,17}$ This and similar systems have been recently studied both experimentally and theoretically. ${ }^{9,16-19}$ In contrast to previous theoretical work, ${ }^{9,19,20}$ our approach fully takes into account the electronic correlations and hybridization of the entire Mn $3 d$ shell. This allows us to get the first complete picture of the Kondo effect and molecular quenching processes in a high-spin complex.

We consider a single MnPc adsorbed at the top site of a $\mathrm{Pb}(111)$ surface as shown in Fig. 1(a). We first relax the atomic structure, orientation, and distance of the molecule to the substrate which is represented by a cluster consisting of five atomic layers. This is done through the common Kohn-Sham (KS) approach to DFT using a standard generalized gradient approximation (GGA) functional ${ }^{21}$ as implemented in the GAUSSIAN09 package. ${ }^{22}$ Next we embed the cluster consisting of the substrate and molecule (hereafter called region $\mathrm{C}$ ) into an effective semi-infinite bulk electrode model as implemented in the code ANT.G, ${ }^{23,24}$ which interfaces GAUSSIAN09. The KS Green's function (GF) of the system $\mathrm{C}$ can now be obtained as $G_{\mathrm{C}}^{0}(\omega)=\left[\omega+\mu-H_{\mathrm{C}}^{0}-\Sigma_{\mathrm{S}}(\omega)\right]^{-1}$, where $H_{\mathrm{C}}^{0}$ is the self-consistent KS Hamiltonian reevaluated considering now $\Sigma_{\mathrm{S}}(\omega)$, which is the embedding self-energy describing the semi-infinite bulk electrode.

To capture many-body effects beyond the DFT level, we have applied the DFT+impurity solver method for 

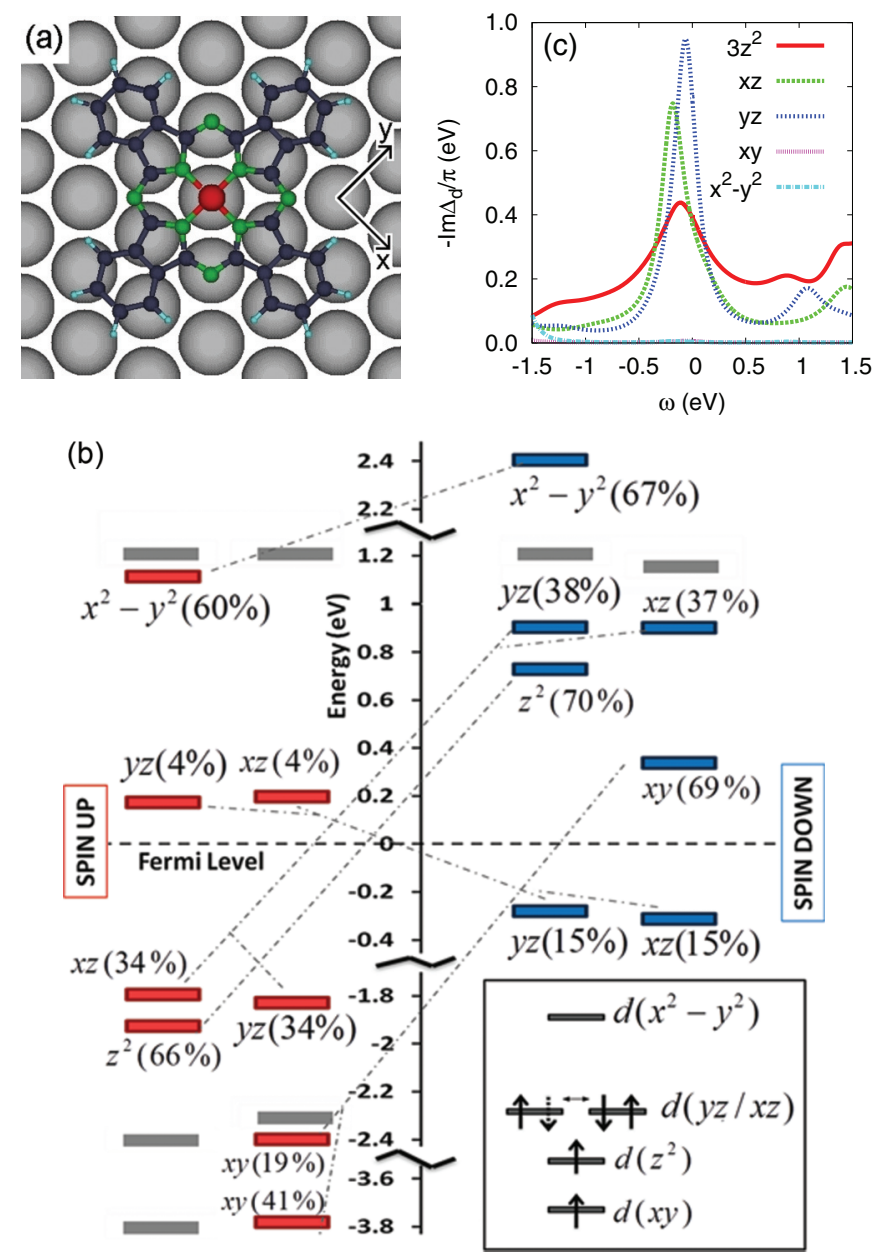

FIG. 1. (Color online) (a) Top view of a PcMn adsorbed on a $\mathrm{Pb}(111)$ surface in its more stable configuration. (b) Molecular orbital energy diagram as obtained from the projected spin-polarized KS Hamiltonian of the adsorbed molecule. The Mn $3 d$ orbital character (percentage) of each molecular orbital is shown. The inset shows the schematics of the Mn $3 d$ orbital energies and associated spins as considered in the OCA calculations. (c) Orbital resolved imaginary part of the hybridization function for the Mn $3 d$ orbitals as obtained from the GGA nonmagnetic electronic structure calculation.

nanoscopic conductors developed by one of us in earlier work. ${ }^{25}$ To this end, the mean-field KS Hamiltonian is augmented by a Hubbard-like interaction term $\hat{\mathcal{H}}_{U}=\sum_{\alpha \beta \gamma \delta \sigma \sigma^{\prime}} U_{\alpha \beta \gamma \delta} d_{\alpha \sigma}^{\dagger} d_{\beta \sigma^{\prime}}^{\dagger} d_{\delta \sigma^{\prime}} d_{\gamma \sigma}$ which accounts for the strongly interacting electrons of the Mn $3 d$ shell. These are different from the bare interactions due to screening processes. The screened Coulomb interaction within the $\mathrm{Mn}$ $3 d$ shell, $U_{\alpha \beta \gamma \delta}$, has been determined using the constrained random phase approximation (RPA) approach. ${ }^{26}$ We find that the matrix elements $U_{\alpha \beta \gamma \delta}$ are somewhat anisotropic with variations of up to $10 \%$ between different orbitals. For the intra-orbital Coulomb repulsion $U_{\alpha \alpha \alpha \alpha}$ we have a mean value of $5.4 \mathrm{eV}$, and for the inter-orbital Coulomb repulsion $U_{\alpha \beta \alpha \beta}(\alpha \neq \beta)$ we have a mean value of $4.1 \mathrm{eV}$. The orbital anisotropy of the direct repulsion will be fully taken into account in our calculations. The exchange matrix elements $U_{\alpha \beta \beta \alpha}$, which give rise to Hund's rule coupling $J_{H}$, also become somewhat orbital-dependent. But here we simply set
$J_{H}$ to the orbital-averaged exchange interaction $J_{H} \equiv\left\langle U_{\alpha \beta \beta \alpha}\right\rangle$ for which we find $0.65 \mathrm{eV}$.

The interacting Mn $3 d$ shell coupled to the rest of the system (organic scaffold + surface) thus constitutes a so-called Anderson impurity model (AIM). The AIM is completely defined by the interaction matrix elements $U_{\alpha \beta \gamma \delta}$, the energy levels $\epsilon_{d}$ of the $3 d$ orbitals, and the so-called hybridization function $\Delta_{d}(\omega)$. The latter describes the (dynamic) coupling of the $\mathrm{Mn}$ $3 d$ shell to the rest of the system and can be obtained from the $\mathrm{KS} \mathrm{GF}^{25}$ as $\Delta_{d}(\omega)=\omega+\mu-\epsilon_{d}^{0}-\left[G_{d}^{0}(\omega)\right]^{-1}$, where $\mu$ is the chemical potential, $\epsilon_{d}^{0}$ are the KS energy levels of the $3 d$ orbitals, and $G_{d}^{0}(\omega)$ is the KS GF projected onto the $3 d$ subspace. The energy levels $\epsilon_{d}$ are obtained from the KS levels, $\epsilon_{d}=\epsilon_{d}^{0}-E_{d c}$ where, as usual in DFT ++ approaches, ${ }^{27}$ a double counting correction (DCC) has to be subtracted to compensate for the overcounting of interaction terms. Here we employ the so-called fully localized or atomic limit DCC, ${ }^{28}$ but generalized to the case of an anisotropic Coulomb repulsion: $E_{d c}^{\alpha}=\sum_{\beta} U_{\alpha \beta \alpha \beta}\left(n_{\beta}-\frac{1}{2 M}\right)-J_{H}\left(N_{3 d}-1\right) / 2$, where $n_{\alpha}$ is the DFT occupation of orbital $\alpha, N_{3 d}$ is the total occupation of the $\mathrm{Mn} 3 d$ shell, and $M$ is the number of correlated orbitals.

The AIM problem is now solved using the one-crossing approximation (OCA).$^{29}$ This yields the electronic self-energy $\Sigma_{d}(\omega)$ which accounts for the electronic correlations of the $3 d$ electrons due to strong electron-electron interactions. The correlated $3 d$ GF is then given by $G_{d}=\left(\left[G_{d}^{0}\right]^{-1}-\Sigma_{d}+\right.$ $\left.E_{d c}\right)^{-1}$. Correspondingly, the correlated $\mathrm{GF}$ for $\mathrm{C}$ is given by $G_{\mathrm{C}}=\left(\left[G_{\mathrm{C}}^{0}\right]^{-1}-\Sigma_{d}+E_{d c}\right)^{-1}$, where $\Sigma_{d}$ and $E_{d c}$ only act within the $3 d$ subspace. From $G_{\mathrm{C}}$ we can calculate the transmission function $T(\omega)=\operatorname{Tr}\left[\Gamma_{\mathrm{T}} G_{\mathrm{C}}^{\dagger} \Gamma_{\mathrm{S}} G_{\mathrm{C}}\right]$, where $\Gamma_{\alpha} \equiv i\left(\Sigma_{\alpha}-\Sigma_{\alpha}^{\dagger}\right)$ for $\alpha=T, S$ describes the coupling of C to the STM tip $(T)$ and to the semi-infinite $\mathrm{Pb}$ surface $(S)$. Since the (small) voltage mainly drops between tip and molecule, the transmission directly yields the differential conductance: $\mathcal{G}(V)=\left(2 e^{2} / h\right) T(e V)$.

The spin-polarized KS spectrum of all molecular orbitals close to the Fermi energy is shown in Fig. 1(b) along with their Mn $3 d$-orbital character. The ones depicted in gray do not have any $\mathrm{Mn}$ atomic character at all, being completely localized on the organic ligand. A strong localization in the $x^{2}-y^{2}, x y$, and $z^{2}$ atomic orbitals is apparent, being signaled by a single molecular orbital (per spin) with strong atomic character. The $x^{2}-y^{2}$ appears as an empty molecular orbital, well above the Fermi level, the charge density in this orbital (see Table I) being only due to the contribution of many molecular orbitals with negligible participation of the atomic orbital. The other two have a localized unpaired electron each. The third unpaired electron is shared between the $x z / y z$ orbitals. The localized character of this spin is masked due to the strong hybridization

TABLE I. Orbital occupations of Mn $3 d$ shell as calculated with DFT on the level of the GGA and with the OCA. Note that the $x^{2}-y^{2}$ orbital was not taken into account in the OCA calculation (see text).

\begin{tabular}{lcccccc}
\hline \hline & $3 z^{2}$ & $x z$ & $y z$ & $x y$ & $x^{2}-y^{2}$ & tot. \\
\hline GGA & 1.19 & 1.18 & 1.19 & 1.04 & 0.67 & 5.27 \\
GGA + OCA & 1.32 & 1.13 & 1.16 & 1.04 & $(0.67)$ & 5.32 \\
\hline \hline
\end{tabular}


of these orbitals with the ligand, there being four molecular orbitals (per spin) with significant $x z / y z$ atomic character.

In Fig. 1(c) we show the imaginary part of the hybridization function $\Delta(\omega)$ which describes the broadening of the Mn $3 d$ levels due to the coupling to the substrate and to the organic part of the molecule. We see that within an energy window of $\pm 1.0 \mathrm{eV}$ around the Fermi level, only three out of the five $3 d$ levels are actually broadened $\left(3 z^{2}, x z, y z\right)$. The $x y$ and $x^{2}-y^{2}$ orbitals, which are parallel to the surface, show no hybridization at all in this energy window. Outside this window (not shown), however, while the $x y$ orbital still does not show any significant coupling, the $x^{2}-y^{2}$-orbital presents a very large peak at $-2.4 \mathrm{eV}$ (and many small peaks), which indicates a strong coupling to the organic ligand. This is actually a manifestation of what crystal-field theory anticipates and the DFT calculation shows, as described in the preceding paragraph.

Since the $x^{2}-y^{2}$ orbital is virtually empty and shifted to high energies, we exclude this orbital from the AIM model from now on. The $3 z^{2}, x z$, and $y z$ orbitals, being the only ones showing hybridization around the Fermi level, are also the only ones susceptible to Kondo screening. All three orbitals feature a strong peak in the hybridization function around the Fermi energy which stem from coupling to molecular orbitals in the organic ligand, which, in turn, couple to the substrate. Note that due to symmetry reasons, the direct coupling of the $x z$ and $y z$ orbitals to the substrate is strongly suppressed in the top position. In contrast, the $3 z^{2}$ orbital also couples to the substrate directly, resulting in a flatter hybridization function.

Let us now turn to the results of the OCA calculation for solving the generalized AIM problem. We find that, except for the $x y$ orbital, the orbitals of the Mn $3 d$ shell are in a mixedvalence state. There are strong fluctuations between a fivefolddegenerate atomic configuration with $N_{d}=4$ electrons and maximal spin $S_{d}=2$ where all orbitals are singly occupied and three fourfold-degenerate atomic configurations with $N_{d}=5$ electrons and $S=3 / 2$ where one of the $3 z^{2}, x z$, and $y z$ orbitals is doubly occupied. This results in an average occupation of the four Mn $3 d$ levels of $N_{d} \approx 4.6$ electrons and an average total spin $\left\langle S_{d}\right\rangle \approx 1.6$ close to $3 / 2$. The extra half-electron stems from the emptied $x^{2}-y^{2}$ orbital and is shared among the $3 z^{2}$, $x z$, or $y z$ orbitals. This leads to strong charge fluctuations in these orbitals (see Table I) and thus quenching of their spin from $3 / 2$ to $\sim 1$. The $x y$ orbital, on the other hand, is essentially singly occupied, thus carrying a spin-1/2. Note that the individual orbital channels are not in a mixed-valence situation as the individual occupations are clearly below 1.5 and therefore the Kondo effect in individual orbitals is possible despite the Mn $3 d$ shell as a whole being in a mixed-valence state. ${ }^{10}$ Although $\left\langle S_{d}\right\rangle$ is finite, the expectation value for any of its projections is zero, since all states with $S_{d}^{z}=-S_{d} \cdots+$ $S_{d}$ contribute equally. Also note that $\left\langle S_{d}\right\rangle \sim 3 / 2$ is not the expectation value of the total spin of the system but only of the Mn $3 d$ shell. The spin of the whole system is lower due to screening by the Kondo effect with the conduction electrons of the substrate and the organic rest of the molecule, as we will see below.

Figure 2(a) shows the spectral function of the Mn $3 d$ shell for different temperatures. We see a sharp Kondo peak developing right at the Fermi level when the temperature is lowered. As can be seen from Fig. 2(b), where we show the orbital-resolved spectral function on a larger energy scale than in Fig. 2(a), the Kondo peak stems from the $3 z^{2}$ orbital, the only orbital directly coupling to the substrate near the Fermi energy. Note that in the existing literature, this orbital is considered to be quenched ${ }^{16}$ and is excluded from correlated models. ${ }^{30}$ The Kondo temperature for this orbital is $T_{K} \sim 100 \mathrm{~K}$. The $x z$ and $y z$ orbitals, on the other hand, each feature small bumps just below the Fermi level with a much larger width $(\sim 0.5 \mathrm{eV})$ than the Kondo peak in the $3 z^{2}$ orbital. We interpret these pronounced peaks in the hybridization function for the $x z$ and $y z$ orbitals as a result of these orbitals only coupling via the organic ligands to the substrate. In other words, these bumps suggest the formation of a many-body singlet state between the Mn $3 d$ level and a molecular orbital in the organic rest of the molecule as in the zero-bandwidth Anderson impurity model (see, e.g., Appendix of Ref. 2). In this model, the formation of the total spin-singlet state between the strongly interacting impurity level and a single noninteracting bath level gives rise to two strongly renormalized resonances below and above the Fermi level. These resonances are precursors of the Kondo peak, which develops as more and more bath levels are added to the model. Therefore, we can think of the spin in the $x z$ and $y z$ orbitals as being screened due to the formation of a many-body singlet state by strong coupling with the organic ligand. The spin-1/2 in the $x y$ orbital, on the other hand, remains unscreened due to a lack of hybridization with the
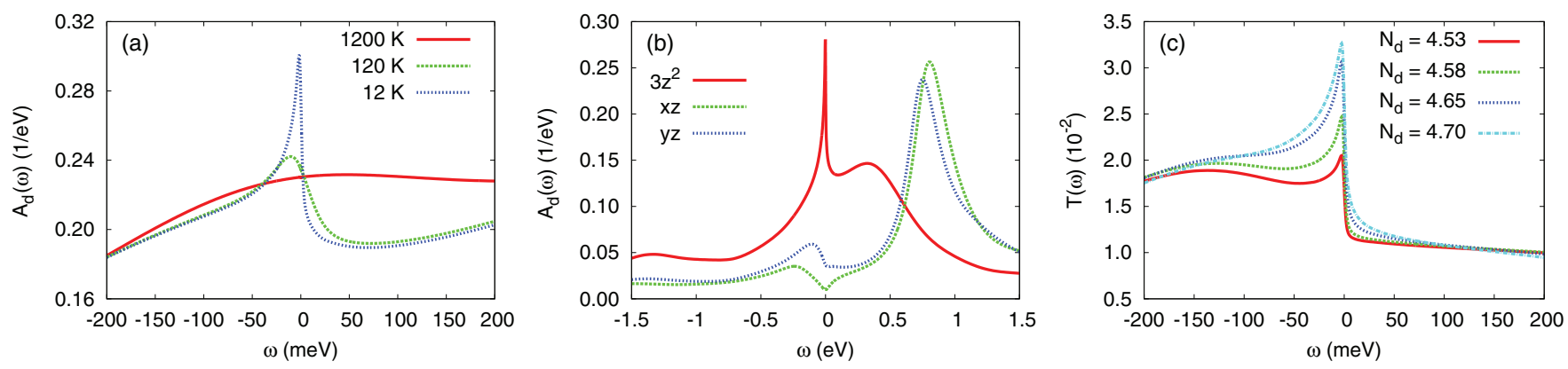

FIG. 2. (Color online) (a) Total spectral function of the Mn $3 d$ shell near the Fermi level for different temperatures. (b) Orbital resolved spectral function of the Mn $3 d$ shell at low temperature $(T \sim 12 \mathrm{~K})$ in a larger energy window than in (a). (c) Transmission function for different filling $N_{d}$ of the four Mn $3 d$ orbitals taken into account in the OCA calculation at low temperature $(T \sim 12 \mathrm{~K})$. 
substrate or molecule near the Fermi level. This is in contrast to existing claims in which this orbital is considered to be screened and responsible for high-energy Kondo features. ${ }^{16}$

Therefore, we are dealing here with an $S=3 / 2$ underscreened Kondo effect in which only the spin $S \approx 1$ within the $3 z^{2}, x z$, and $y z$ orbitals is screened, leaving a residual spin-1/2 in the Mn $x y$ orbital which may lead to so-called singular Fermi-liquid behavior. ${ }^{31}$ Only the screening of the spin within the $3 z^{2}$ orbital gives rise to a Kondo resonance, while no significant low-bias experimental signatures are expected from the strongly coupled spin in the $x z / y z$ orbitals. Since the Kondo temperature of the $3 z^{2}$ channel is too high, it is not unrealistic to attribute the Shiba peaks in Ref. 17 to a low-energy scale Kondo screening of the $x y$ orbital in a lower-symmetry experimental situation. ${ }^{30}$

The Kondo resonance appearing in the spectral function of the Mn $3 d$ shell for low temperatures is somewhat asymmetric. This is mainly a result of two effects: On the one hand, charge fluctuations in the $3 z^{2}$ orbital make the Kondo peak asymmetric due to the proximity of the upper Hubbard peak. On the other hand, the modulation of the hybridization function due to the coupling to the organic ligands near the Fermi level further enhances this asymmetry. The bumps in the spectral function of the $x z$ and $y z$ orbitals, on the other hand, do not have a significant contribution to the asymmetry of the Kondo peak due to their small spectral weight. This asymmetry of the Kondo peak in the spectral function is even more enhanced in the tunneling spectra, as can be seen in Fig. 2(c), where we show the tunnel transmission $T(\omega)$ calculated for a $\mathrm{Pb}$ tip positioned above the $\mathrm{Mn}$ atom at a distance of $5 \AA$. The reason for this further enhancement is the modulation of the Mn $3 d$ spectral function by the DOS of the $\mathrm{Pb}$ tip and the $\mathrm{Pb}$ substrate. For a Au tip we actually find that the tunnel spectra (not shown) are a little bit less asymmetric. Therefore, the peak in the tunnel spectra just stems from the Kondo peak in the $3 z^{2}$ orbital. In fact, a sharp Kondo peak in either the $x z$ or the $y z$ channel would rather give rise to a dip in the tunneling, but not to a peak since the direct tunnel matrix elements between the tip and these two orbitals vanish for symmetry reasons.
Charge fluctuations usually have a strong effect on the Kondo screening. In Fig. 2(c), we show the effect of altering the occupation of the Mn $3 d$ shell on the tunneling spectra by shifting the Mn $3 d$ levels by a few decimal eV. We see that the shape of the Kondo resonance, and in particular its width, is strongly affected by the slight changes in the occupation of the Mn $3 d$ shell. In fact, our calculated line shapes reproduce very well the variation of line shapes measured in recent experiments. Hence we conclude that the experimentally observed variation in line shapes for different PcMn molecules on the $\mathrm{Pb}(111)$ surface ${ }^{16,17}$ is likely due to slight changes in the occupation of the Mn $3 d$ shell induced by slight variations in the structure or environment of the molecule in the experiments.

In summary, we have studied the correlated electronic structure of a MnPc adsorbed on the $\mathrm{Pb}(111)$ surface, fully taking into account the strong electronic correlations originating from the Mn $3 d$ shell. Our results show that the adsorption does not essentially modify the total spin $S=3 / 2$ of the molecule, which is distributed among four of the five $3 d$ orbitals. This finding is in stark contrast to previous works which assume or find a spin-1 (Ref. 30) or even a spin-1/2 state due to strong quenching with the substrate and organic ligand. ${ }^{16}$ We further find that the experimentally observed asymmetric Kondo resonance in this system ${ }^{16,17}$ is due to an underscreened Kondo effect in which a spin-1/2 in the Mn $3 d$ shell remains unscreened. The Kondo resonance in the tunnel spectra actually stems from only one of the Kondo-screened orbitals. Its peculiar line shape arises from the modulation of the hybridization function due to strong coupling to the organic ligand, not being necessary to invoke the superposition of two Kondo peaks with different Kondo temperatures, as was done in Ref. 17.

This work was supported by MICINN under Grants No. FIS2010-21883 and No. CONSOLIDER CSD2007-0010. M.S. acknowledges computational support from the CCC of the Universidad Autónoma de Madrid. We are also grateful to $\mathrm{K}$. Haule for providing us with the OCA impurity solver. *djacob@mpi-halle.de

${ }^{1}$ J. Kondo, Prog. Theor. Phys. 32, 37 (1964).

${ }^{2}$ A. C. Hewson, The Kondo Problem to Heavy Fermions (Cambridge University Press, Cambridge, 1997).

${ }^{3}$ V. Madhavan, W. Chen, T. Jamneala, M. F. Crommie, and N. S. Wingreen, Science 280, 567 (1998); J. Li, W.-D. Schneider, R. Berndt, and B. Delley, Phys. Rev. Lett. 80, 2893 (1998); N. Knorr, M. A. Schneider, L. Diekhöner, P. Wahl, and K. Kern, ibid. 88, 096804 (2002); L. H. Yu, Z. K. Keane, J. W. Ciszek, L. Cheng, J. M. Tour, T. Baruah, M. R. Pederson, and D. Natelson, ibid. 95, 256803 (2005); M. Ternes, A. J. Heinrich, and W.-D. Schneider, J. Phys.: Condens. Matter 21, 053001 (2009).

${ }^{4}$ D. Gatteschi, R. Sessoli, and J. Villain, Molecular Nanomagnets (Oxford University Press, Oxford, 2006).

${ }^{5}$ S. A. Wolf, D. D. Awschalom, R. A. Buhrman, J. M. Daughton, S. von Molnár, M. L. Roukes, A. Y. Chtchelkanova, and D. M. Treger, Science 294, 1488 (2001).
${ }^{6}$ V. Iancu, A. Deshpande, and S.-W. Hla, Nano Lett. 6, 820 (2006); T. Choi, S. Bedwani, A. Rochefort, C.-Y. Chen, A. J. Epstein, and J. A. Gupta, ibid. 10, 4175 (2010).

${ }^{7}$ J. J. Parks, A. R. Champagne, T. A. Costi, W. W. Shum, A. N. Pasupathy, E. Neuscamman, S. Flores-Torres, P. S. Cornaglia, A. A. Aligia, C. A. Balseiro, G. K.-L. Chan, H. D. Abruña, and D. C. Ralph, Science 328, 1370 (2010).

${ }^{8}$ A. Zhao, Q. Li, L. Chen, H. Xiang, W. Wang, S. Pan, B. Wang, X. Xiao, J. Yang, J. G. Hou, and Q. Zhu, Science 309, 1542 (2005).

${ }^{9}$ A. Strozecka, M. Soriano, J. I. Pascual, and J. J. Palacios, Phys. Rev. Lett. 109, 147202 (2012); L. Liu, K. Yang, Y. Jiang, B. Song, W. Xiao, L. Li, H. Zhou, Y. Wang, S. Du, M. Ouyang, W. A. Hofer, A. H. Castro Neto, and H.-J. Gao, Sci. Rep. 3, 1 (2013).

${ }^{10} \mathrm{P}$. Noziéres and A. Blandin, J. Phys. 41, 193 (1980).

${ }^{11}$ N. Roch, S. Florens, T. A. Costi, W. Wernsdorfer, and F. Balestro, Phys. Rev. Lett. 103, 197202 (2009). 
${ }^{12}$ S. Di Napoli, A. Weichselbaum, P. Roura-Bas, A. A. Aligia, Y. Mokrousov, and S. Blügel, Phys. Rev. Lett. 110, 196402 (2013).

${ }^{13}$ B. E. Williamson, T. C. Van Cott, M. E. Boyle, G. C. Misener, M. J. Stillman, and P. N. Schatz, J. Am. Chem. Soc. 114, 2412 (1992).

${ }^{14}$ J. H. Van Vleck, Phys. Rev. 41, 208 (1932); C. E. Housecroft and A. G. Sharpe, Inorganic Chemistry (Prentice Hall, Englewood Cliffs, NJ, 2004).

${ }^{15}$ S. Stepanow, P. S. Miedema, A. Mugarza, G. Ceballos, P. Moras, J. C. Cezar, C. Carbone, F. M. F. de Groot, and P. Gambardella, Phys. Rev. B 83, 220401 (2011).

${ }^{16}$ Y.-S. Fu, S.-H. Ji, X. Chen, X.-C. Ma, R. Wu, C.-C. Wang, W.-H. Duan, X.-H. Qiu, B. Sun, P. Zhang, J.-F. Jia, and Q.-K. Xue, Phys. Rev. Lett. 99, 256601 (2007).

${ }^{17}$ K. J. Franke, G. Schulze, and J. I. Pacual, Science 332, 940 (2011).

${ }^{18}$ L. Gao, W. Ji, Y. B. Hu, Z. H. Cheng, Z. T. Deng, Q. Liu, N. Jiang, X. Lin, W. Guo, S. X. Du, W. A. Hofer, X. C. Xie, and H.-J. Gao, Phys. Rev. Lett. 99, 106402 (2007).

${ }^{19}$ E. Minamitani, N. Tsukahara, D. Matsunaka, Y. Kim, N. Takagi, and M. Kawai, Phys. Rev. Lett. 109, 086602 (2012).
${ }^{20}$ L. G. G. V. Dias da Silva, M. L. Tiago, S. E. Ulloa, F. A. Reboredo, and E. Dagotto, Phys. Rev. B 80, 155443 (2009); R. Korytár and N. Lorente, J. Phys.: Condens. Matter 23, 355009 (2011).

${ }^{21}$ A. D. Becke, Phys. Rev. A 38, 3098 (1988).

${ }^{22}$ M. J. Frisch et al., Gaussian 09 Revision A.l (Gaussian Inc., Wallingford, CT, 2009).

${ }^{23}$ J. J. Palacios, D. Jacob et al., ALACANT software package.

${ }^{24}$ D. Jacob and J. J. Palacios, J. Chem. Phys. 134, 044118 (2011).

${ }^{25}$ D. Jacob, K. Haule, and G. Kotliar, Phys. Rev. Lett. 103, 016803 (2009); D. Jacob and G. Kotliar, Phys. Rev. B 82, 085423 (2010).

${ }^{26}$ F. Aryasetiawan, M. Imada, A. Georges, G. Kotliar, S. Biermann, and A. I. Lichtenstein, Phys. Rev. B 70, 195104 (2004).

${ }^{27}$ A. I. Lichtenstein and M. I. Katsnelson, Phys. Rev. B 57, 6884 (1998).

${ }^{28}$ M. T. Czyzyk and G. A. Sawatzky, Phys. Rev. B 49, 14211 (1994).

${ }^{29}$ K. Haule, S. Kirchner, J. Kroha, and P. Wölfle, Phys. Rev. B 64, 155111 (2001).

${ }^{30}$ J. Bauer, J. I. Pascual, and K. J. Franke, Phys. Rev. B 87, 075125 (2013).

${ }^{31}$ P. Coleman and C. Pépin, Phys. Rev. B 68, 220405 (2003). 